\title{
Genetic testing and first presymptomatic diagnosis in Moroccan families at high risk for breast/ovarian cancer
}

\author{
FATIMA ZAHRA LAARABI ${ }^{1,2}$, IMANE CHERKAOUI JAOUAD ${ }^{1,2}$, KARIM OULDIM $^{2}$, \\ NISRINE ABOUSSAIR ${ }^{2}$, ABDELOUAHED JALIL $^{3}$, BRAHIM EL KHALIL EL GUEDDARI ${ }^{4}$, \\ NOUREDDINE BENJAAFAR ${ }^{4}$ and ABDELAZIZ SEFIANI ${ }^{1,2}$ \\ ${ }^{1}$ Centre de Génomique Humaine, Université Mohamed V Souissi; ${ }^{2}$ Département de Génétique Médicale, \\ Institut National d'Hygiène; ${ }^{3}$ Service de Chirurgie Oncologique; ${ }^{4}$ Service de Radiothérapie, \\ Institut National d'Oncologie, Rabat, Morocco
}

Received October 19, 2010; Accepted January 17, 2011

DOI: $10.3892 / \mathrm{ol} .2011 .248$

\begin{abstract}
Germline mutations in the BRCAl and $B R C A 2$ genes highly predispose to breast and ovarian cancers and are responsible for a substantial proportion of familial breast and ovarian cancers. No female individuals from families from Morocco affected by breast cancer with mutations of these genes have previously been reported, and clinicians in Morocco are unaccustomed to dealing with healthy female individuals carrying mutations in the BRCA genes. This study aimed to report the initial experience of a group of Moroccan investigators carrying out predictive genetic testing to detect a known familial mutation in healthy Moroccan females with a high risk of developing breast cancer and to introduce supervision of these asymptomatic female carriers as a new approach in the prevention and early diagnosis of breast and ovarian cancers in Morocco. Presymptomatic diagnosis was carried out using DNA genetic testing in 5 healthy Moroccan female individuals from three families with an elevated risk of developing breast cancer. These are the first Moroccan families reported to be affected by breast cancers associated with BRCA mutations. Presymptomatic diagnosis was carried out for breast cancer in 5 female individuals from three Moroccan families with BRCA mutations. Two of the families are the first reported incidence of the founder mutation Ashkenazi BRCA1-185_186delAG in Moroccan patients. The third family carried the known BRCA2 mutation c.5073dupA/p. trp1692metfsX3. We tested the presence of these mutations in 5 asymptomatic healthy females from the three families. Two sisters from family 1 carried the BRCA1-185_186delAG mutation, whereas the third female individual from family 2
\end{abstract}

Correspondence to: Professor Abdelaziz Sefiani, Département de Génétique Médicale, Institut National d'Hygiène 27, Avenue Ibn Battouta, BP 769, Rabat, Morocco

E-mail: sefianigen@hotmail.com

Key words: BRCA1/2 genes, hereditary breast-ovarian cancer, presymptomatic DNA testing carried the c.5073dupA/p.trp1692metfsX3 mutation. However, one healthy female individual and her mother from family 3 did not carry the familial mutation of the BRCAl gene. This study found BRCA mutations in three asymptomatic subjects, suggesting that this is the first step towards the development of persistent medical monitoring of females from families with a history of breast and ovarian cancers. Consequently, it is crucial for oncologists in Morocco to initiate the supervision of healthy female individuals with genetic defects which may lead to hereditary cancers.

\section{Introduction}

Tumours and cancers have become major public health problems in Morocco, particularly due to a rapid epidemiological transition. Cancer mortality remains high since diagnosis is frequently delayed, and medical management is difficult and costly. Health authorities in Morocco launched various campaigns to combat cancer, since a large proportion of these diseases may be prevented or detected early and properly treated. However, familial forms of cancer only recently attracted interest, and some local genetic centres have begun to provide oncogenetic services and offer genetic testing for certain inherited forms of cancer with special focus on breast cancer.

Hereditary breast and ovarian cancer syndrome is an inherited cancer-susceptibility syndrome. This syndrome is characterised by multiple family members with breast or ovarian cancer or both, the presence of both breast and ovarian cancer in a single individual, and early age of breast cancer onset (1). Germline mutations in BRCA1 and BRCA2 genes account for a substantial proportion of families with hereditary breast and ovarian cancer syndrome. Approximately $10 \%$ of cases of ovarian cancer and 3-5\% of cases of breast cancer are known to be associated with germline mutations in BRCA1 and BRCA2 (2-4). The two genes are tumoursuppressor genes, and complete loss of their wild-type allele is a common mechanism of gene inactivation in tumourigenesis (5). BRCA1 and BRCA2 are involved in pathways that are crucial for DNA damage recognition, double-strand 
Table I. Clinical details of the three probands in the three families.

\begin{tabular}{llll}
\hline & Proband III-8/family 1 & Proband III-18/family 2 & Proband III-17/family 3 \\
\hline Age (years) & 37 & 48 & 55 \\
Bilateral breast cancer & No & No & No \\
Left breast cancer & No & Yes & No \\
First menstrual period (years) & 13 & 13 & 14 \\
Tobacco, alcohol use & None & None & \\
Menopause & No & No & Yes \\
Tumour size (cm) & 8 & 1 & 1.8 \\
Histological diagnosis & Ductal carcinoma & Ductal carcinoma & Ductal carcinoma \\
Inflammatory breast cancer & No & No & No \\
SBR & III & II & III \\
Progesterone receptor & Positive & Positive & Positive \\
Oestrogen receptor & Positive & Positive & Positive \\
TNM stage & T3N0M0 & T1N0M0 0 \\
Surgery & Radical mastectomy & Tumourectomy & T2N1M0 \\
& Axillary lymph node & Axillary lymph node & Radical mastectomy \\
& dissection & dissection & Axillary lymph node \\
Radiotherapy & Yes & Yes & Yes \\
Chemotherapy & FEC & CMF & 3 FEC + taxotere \\
Clinical response (3 months) & Complete response & Complete response & Complete response \\
Clinical response (6 months) & Complete response & Liver metastasis & Complete response
\end{tabular}

aFluorouracil, epirubicin, cyclophosphamide; ${ }^{b}$ cyclophosphamide, methotrexate, fluorouracil. SBR, Scarff-Bloom-Richardson system.

break repair, checkpoint control, transcription regulation and chromatin remodeling (6). Mutations in BRCA genes lead to defects in DNA repair processes resulting in elevated genome instability and a predisposition to breast and ovarian cancers.

BRCA1 and BRCA2 are large genes containing 5,592 and 11,385 nucleotides spread over approximately 100,000 bases of genomic DNA each. More than 1,000 BRCA mutations are reported in the Breast Cancer Information Core database (7). In the general population, it is estimated that approximately 1 in 300 to 1 in 800 individuals carry a mutation in the BRCA1 or BRCA2 gene (8).

Hereditary breast and ovarian cancer syndrome is characterised by four or more breast or ovarian cancers within a family, typically occurring at young ages or bilaterally in the case of breast cancer. Ovarian cancers are typically epithelial, with a younger age of onset in BRCAl carriers (mean 54 years), compared to sporadic ovarian cancers (mean 63 years), which have a comparable age of onset to BRCA2 carriers (mean 62 years). Breast cancer in these families is also typically noted at younger ages, particularly in BRCAl carriers, compared to that in the general population (9).

Clinical genetic testing for gene mutations allows physicians to identify more precisely female individuals who are at a substantial risk of developing breast and ovarian cancers. For these individuals, screening and prevention strategies can be instituted to reduce the risks (1).

The main objective of this study is to report the first use of predictive genetic testing to detect a known familial mutation in healthy Moroccan females with a high risk of developing breast cancer and introduce supervision of these asymptomatic female carriers as a new aspect in the combat against breast and ovarian cancers in Morocco.

\section{Patients and methods}

Patients and their family members. Patients with breast cancer from unrelated families were referred to our Medical Genetics Department for genetic counseling. BRCA genetic testing was carried out in patients with early age of onset at diagnosis, positive family history or bilateral breast cancer. Table I shows the clinical data for three index cases from 23 unrelated families enrolled in the study. Genetic testing for presymptomatic diagnosis was requested by 5 healthy female individuals among 45 female individuals with an elevated risk of breast cancer from the three families in whom mutations of the BRCA1/2 genes were identified (Fig. 1). The study was approved by our local ethics committee, and the subjects included in the study gave written informed consent for DNA testing.

Mutation screening. Genomic DNA was extracted from peripheral blood lymphocytes using salt extraction methods and a complete analysis of BRCA1/2 coding regions by bi-directional sequencing was performed. When BRCA mutations were detected, they were confirmed on a second patient DNA sample. The familial mutation was identified by targeted direct sequencing of DNA from family members who were at a high risk of developing breast or ovarian cancers according to their pedigree data and who had requested this type of genetic testing. 
A

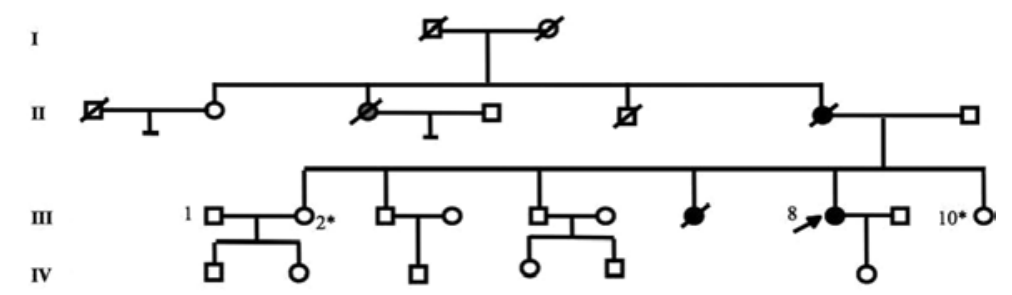

Ovary

Breast

B

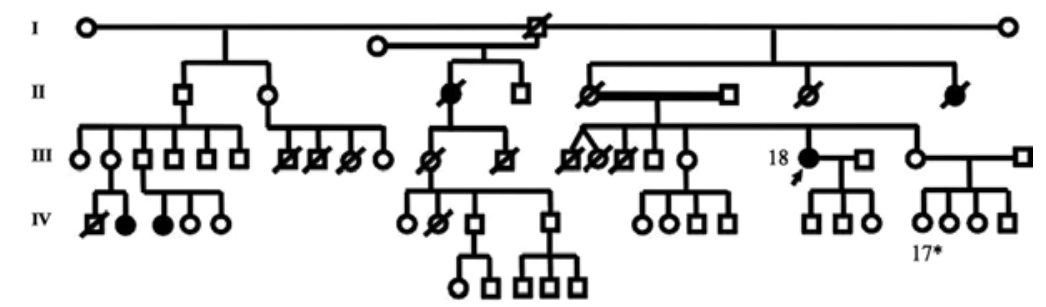

C

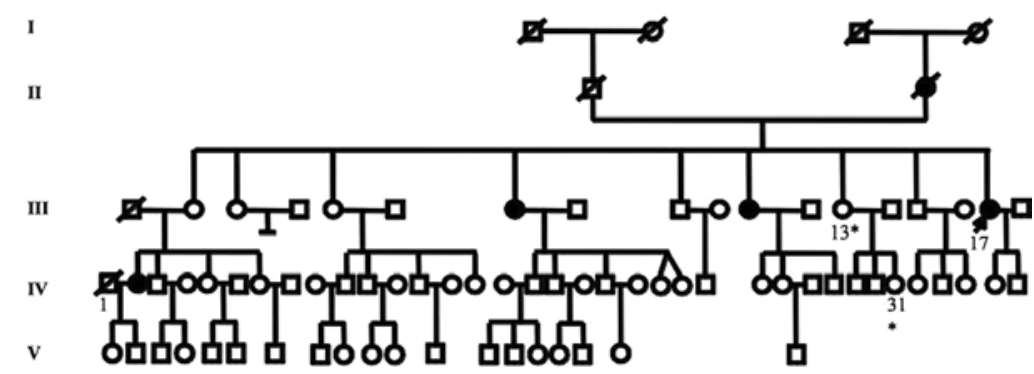

Figure 1. Pedigree of the three families with mutation. (A) Family 1; (B) family 2 and (C) family 3. "Patients who requested presymptomatic diagnosis.

\section{Results}

BRCA1/2 mutation analysis. Two BRCA mutations were identified in the three families. The 185delAG (c.68_69delAG) mutation in exon 2 of the BRCAl gene was found in two unrelated families and the c.5073dupA; p.Trp1692MetfsX3 mutation in exon 11 of the BRCA2 gene was detected in one family. The finding of these mutations in the three families enabled us to conduct predictive DNA testing for 5 female individuals who requested evaluation of their genetic status with regards to their familial BRCA mutation.

Two sisters from family 1 (III-2 and III-10; Fig. 1A) were found to be carriers of the BRCA1-185delAG mutation. Clinical and imaging surveillance of the two sisters detected a breast tumour using magnetic resonance imaging of the breast in the 48-year-old sister (III-2), currently living abroad and followed by a foreign medical team. Additionally, ovarian cancer was detected in her 42-year-old sister (III-10) living in Morocco by transvaginal pelvic ultrasound with colour Doppler. This patient underwent salpingo-oophorectomy, and the histology revealed a papillary serous carcinoma, stage I, T2NOM0. She was treated with six adjuvant cycles of chemotherapy type taxol-carboplatin. A complete response was achieved.

In family 2, Patient IV-17 (Fig. 1B) was a carrier of the familial mutation c.5073dupA/p.Trp1692MetfsX3 in exon 11 of the BRCA2 gene, confirmed by DNA testing. Her mother (III-20) was identified as an obligate carrier. These two female patients are healthy with no cancer history and are under surveillance according to standard protocols (clinical breast examination semi-annually, annual mammography and breast magnetic resonance imaging screening, and annual transvaginal pelvic ultrasound with colour Doppler).

In family 3 , genetic testing showed that the female patient identified as IV-31, and her mother (III-13; Fig. 1C) were not carriers of the familial BRCA1-185delAG mutation. The female individuals were informed that their risk of developing breast cancer does not differ from females of the same age in the general population.

\section{Discussion}

Germline mutations in either of the two predisposing genes, $B R C A 1$ and BRCA2, account for a significant proportion of hereditary breast and ovarian cancers. Approximately $10 \%$ of cases of ovarian cancer and 3-5\% of cases of breast cancer are due to germline mutations in BRCAl and BRCA2 (2). In the general population, it is estimated that approximately 1 in 300 to 1 in 800 individuals carry a mutation in BRCA1 or BRCA2 (3).

A large number of different mutations in the two genes have been reported, some of which are founder mutations noted in the ethnic groups of Ashkenazi Jews, Icelanders, the French and other populations $(10,11)$. Three founder mutations have been identified in Ashkenazi Jews, BRCA1-185delAG, BRCA1-5382insC and 6174delT in the BRCA2 gene. The overall 
rate of the three founder mutations is $2.6 \%(1 / 40)$ compared to the rate of $0.2 \%(1 / 500)$ of mutation carriers in BRCA1/2 in the general population $(12,13)$. The three mutations of BRCA1185delAG, BRCA1-5382insC and BRCA2-6174delT were found in $1,0.13$ and $1.52 \%$ of the Ashkenazi Jews population, respectively $(12,13)$.

Among the North African population, the mutational spectrum of BRCA1/2 has yet to be well characterised. Mutation c.798_799delTT has been observed in two Algerian families, as well as in two Tunisian families presenting with breast cancer, indicating the first non-Jewish founder mutation to be described in Northern Africa (14).

In the present study, the 185 delAG mutation in exon 2 of the BRCA1 gene and the c.5073dupA mutation in exon 11 of the $B R C A 2$ gene are the first mutations described in Moroccan families with hereditary breast and ovarian cancer syndrome. The 185delAG mutation in exon 2 of the BRCA1 gene was detected in families 1 and 3, whereas the proband III-8 in family 2 was a carrier of the c.5073dupA/p.Trp1692MetfsX3 mutation in exon 11 of the BRCA2 gene. Genetic counseling was offered to each of the families. Female individuals with either BRCA mutation have a cumulative lifetime risk of invasive breast cancer of $65-74 \%(15,16)$. For females with a BRCA1 mutation, the risk of ovarian cancer is $39-46 \%$. This risk is lower (12-20\%) for women carrying BRCA2 mutations $(15,16)$.

The 185delAG mutation reported for the first time in females of two Moroccan families may be common in Morocco. Thus, further investigations into this mutation should be conducted in a larger cohort of patients. If the repeated occurrence of the $185 \mathrm{del}$ AG mutation is confirmed in the Moroccan population, this may simplify genetic testing and have clinical and public health implications.

The finding of the mutations in the three families enabled us to conduct DNA testing in 5 female individuals to determine whether they had inherited the familial BRCA mutation. Once a mutation was identified in the proband III- 8 in family 1 , the genetic status of two asymptomatic sisters was determined. The two sisters were found to be carriers of the familial BRCA1-185delAG mutation. Therefore, a medical follow-up of the two sisters was implemented. Surveillance imaging detected breast cancer by magnetic resonance imaging of the breast in a 48-year-old female (Patient III-2; Fig. 1A), and ovarian cancer was detected by transvaginal pelvic ultrasound with colour Doppler in her 42-year-old sister (Patient III-10; Fig. 1A); a complete response to treatment was achieved. In family 2, patient IV-17 (Fig. 1B) was a carrier of the familial mutation c.5073dupA/p.Trp1692MetfsX3 mutation of BRCA2. Consequently, her mother was identified as an obligate carrier. The two patients are healthy thus far, with no history of cancer, and are under supervision in accordance with standard protocols. This BRCA2 mutation confers an increased risk of developing breast or ovarian cancers depending on age and the penetrance of the mutation.

In family 3, genetic testing identified that patient IV-31 (Fig. 1C) and her mother were not carriers of the familial $B R C A 1$ mutation. Consequenlty, it was determined that the risk of developing sporadic breast cancer is at a rate roughly equivalent to that of the general population. These female patients may have undergone unnecessary intervention, including prophylactic surgery. Thus, the availability of the BRCA analysis has beneficial impact on the care and counseling of female individuals at risk. Once a mutation is identified in an affected person, asymptomatic family members can subsequently be tested for this mutation, since first-degree relatives of patients with breast cancer have increased risk for early onset of this disease (17). Female individuals who remain untested for the gene mutation, but have a first-degree relative with a BRCA mutation, have a 36-85\% lifetime risk of developing breast cancer, indicating a need for accurate, life-long surveillance. The American Cancer Society and the National Comprehensive Cancer Network recommend that magnetic resonance imaging be used in addition to, and not instead of mammography, this surveillance (18).

Genetic testing can be extremely valuable in the clinical process and for personal decision-making for high-risk families. The identification of a carrier of a BRCA mutation in a family is useful in the clinical management of the affected patient, but also provides appropriate recommendations for other members of the family. The identification of a family that is highly susceptible to hereditary cancer syndrome is the key to management of these families.

In conclusion, we report our initial experience in the management and presymptomatic diagnosis of breast cancer in three Moroccan families with hereditary breast and ovarian cancer syndrome and known BRCA1/2 gene mutations. Efforts are underway to reduce the high incidence and mortality associated with breast cancer on a global scale. Morocco, which has 30,000 new cases of cancer every year, has launched an ambitious national program of prevention and monitoring of cancer in the population for the decade 2010-2019. This program is based on the early detection of female individuals at high risk and should also integrate molecular testing and management of females with an increased familial risk of breast and ovarian cancers, since genetic predisposition is the strongest risk factor. Early detection is of high priority in the medical management of this diseasein order for recommendations to be made for public health action on molecular genetic testing and genetic counseling.

\section{Acknowledgements}

The authors extend their sincere thanks to all of the staff of the Department of Medical Genetics of the National Institute of Health for their continuous support. We are particularly thankful to Dr Rachid Tazi Ahnini for the indispensable editorial assistance.

\section{References}

1. ACOG Committee on Practice Bulletins: Hereditary breast and ovarian cancer syndrome. Gynecol Oncol 113: 6-11, 2009.

2. Robson ME, Boyd J, Borgen PI and Cody HS III: Hereditary breast cancer. Curr Probl Surg 38: 387-480, 2001.

3. Risch HA, McLaughlin JR, Cole DE, et al: Population BRCA1 and BRCA2 mutation frequencies and cancer penetrances: a kin-cohort study in Ontario, Canada. J Natl Cancer Inst 98: 1694-1706, 2006.

4. Rubin SC, Blackwood MA, Bandera C, et al: BRCA1, BRCA2, and hereditary nonpolyposis colorectal cancer gene mutations in an unselected ovarian cancer population: relationship to family history and implications for genetic testing. Am J Obstet Gynecol 178: 670-677, 1998. 
5. Ratajska M, Brozek I, Senkus-Konefka E, Jassem J, Stepnowska M, Palomba G, Pisano M, Casula M, Palmieri G, Borg A and Limon J: BRCA1 and BRCA2 point mutations and large rearrangements in breast and ovarian cancer families in Northern Poland. Oncol Rep 19: 263-268, 2008.

6. Mohamad HB: Counseling for male BRCA mutation carriers: a review. Apffelstaedt JP Breast 17: 441-450, 2008.

7. Filippini S, Blanco A, Fernández-Marmiesse A, AlvarezIglesias V, Ruíz-Ponte C, Carracedo A and Vega A: Multiplex SNaPshot for detection of BRCA1/2 common mutations in Spanish and Spanish related breast/ovarian cancer families. BMC Med Genet 29: 40, 2007.

8. Whittemore AS, Gong G and Itnyre J: Prevalence and contribution of BRCA1 mutations in breast cancer and ovarian cancer: results from three U.S. population-based casecontrol studies of ovarian cancer. Am J Hum Genet 60: 496-504, 1997.

9. Hanson $\mathrm{H}$ and Hodgson S: Cancer genetics and reproduction, best practice and research. Clin Obstet Gynaecol 24: 3-18, 2010.

10. Neuhausen SL: Ethnic differences in cancer risk resulting from genetic variation. Cancer 86: 2575-2582, 1999.

11. Ferla R, Calo V, Cascio S, Rinaldi G, Badalamenti G, Carreca I, Surmacz E, Colucci G, Bazan V and Russo A: Founder mutations in BRCA1 and BRCA2 genes. Ann Oncol 18: 93-98, 2007.

12. Roa BB, Boyd AA, Volcik K, et al: Ashkenazi Jewish population frequencies for common mutations in BRCA1 and BRCA2. Nat Genet 14: 185-187, 1996.
13. Neuhausen S, Gilewski T, Norton L, et al: Recurrent BRCA2 6174delT mutations in Ashkenazi Jewish women affected by breast cancer. Nat Genet 13: 126-128, 1996.

14. Uhrhammer N, Abdelouahab A, Lafarge L, Feillel V, Ben Dib A and Bignon YJ: BRCA1 mutations in Algerian breast cancer patients: high frequency in young, sporadic cases. Int $\mathrm{J}$ Med Sci 5: 197-202, 2008.

15. Antoniou A, Pharoah PD, Narod S, et al: Average risks of breast and ovarian cancer associated with BRCA1 or BRCA2 mutations detected in case series unselected for family history: a combined analysis of 22 studies. Am J Hum Genet 72: 1117-1130, 2003.

16. King MC, Marks JH and Mandell JB: Breast and ovarian cancer risks due to inherited mutations in BRCA1 and BRCA2. New York Breast Cancer Study Group. Science 302: 643-646, 2003.

17. Loman N, Bladstrom A, Johannsson O, Borg A and Osson H: Cancer incidence in relatives of a population-based set of cases of early-onset breast cancer with a known BRCA1 and BRCA2 mutation status. Breast Cancer Res 5: 175-186, 2003.

18. Waters CM, Hoover AC, McClain LC, Moore TT, Rogers CT and Thornton K: Current guidelines and best practice evidence for intensified/enhanced breast cancer screening in women with BRCA mutations. J Nurs Pract 5: 447-453, 2009. 